\title{
Very Long Baseline Interferometry observations: the closest look at the cores of AGN
}

\author{
Marcello Giroletti ${ }^{1}$, Rocco Lico ${ }^{1,2}$, \\ Kazuhiro Hada ${ }^{1}$ and Gabriele Giovannini ${ }^{1,2}$ \\ ${ }^{1}$ INAF Istituto di Radioastronomia, 40129 Bologna, Italy \\ ${ }^{2}$ Dept. of Physics and Astronomy, Univ. of Bologna \\ email: giroletti@ira.inaf.it
}

\begin{abstract}
Very Long Baseline Interferometry (VLBI) provides the highest angular resolution achievable in astronomy, reaching sub-milliarcsecond scales. For radio loud AGNs, this offers the unique opportunity to directly image and monitor the fine details of the jet structure, approaching the event horizon in the nearest and most supermassive black holes, like in M87. After a quick review of various VLBI facilities, we present results from recent VLBI monitoring projects on two remarkable radio and high energy sources: the radio galaxy M87 and the BL Lac object Mrk421. For the latter, we present a detailed analysis of the jet structure in total intensity and polarization through the whole 2011, during which a multi-wavelength campaign took place. We reveal flux density variability but no significant changes in the jet structure. In M87, we detect and follow the evolution of the core and of the jet feature HST-1; we reveal superluminal motion of components within HST-1, with a possible connection between ejection of new such components and the occurrence of very high energy flares. Estimates on the physical parameters for both sources are given (Doppler factor, viewing angle, magnetic field, etc.).
\end{abstract}

Keywords. techniques: high angular resolution, radio continuum: galaxies, galaxies: nuclei, BL Lacertae objects: individual (Mrk421), galaxies: individual (M87)

\section{Introduction}

Radio waves travel relatively undisturbed through the Earth's atmosphere, so that interferometry is possible on very long baselines. The resolution of an interferometer is given by $\theta=\lambda / B$, where $\lambda$ is the observing wavelength and $B$ the length of the (maximum) baseline. For Very Long Baseline Interferometry (VLBI), stations spread over different countries allow us to currently obtain angular resolutions of up to a few tens of microarcsecond, well beyond those achievable in any other frequency. As a reference, we remind that $10 \mu$ as $\sim 100 R_{\text {Sch }}$ for a $10^{9} M_{\odot}$ supermassive black hole $(\mathrm{SMBH})$ at a redshift $z=0.05$, and 1 mas $\sim 7$ pc at $z=3$.

Traditionally, VLBI studies of Active Galactic Nuclei (AGN) have been limited to radio loud (RL) sources, the only systems reaching brightness temperatures high enough to be revealed with the narrow beam and the limited sensitivity of VLBI arrays. However, recent sensitivity improvements provided by an increase in number of participating stations, quality of the receivers, and correlated bandwidth have extended the realm of VLBI also to radio quiet (RQ) AGNs (e.g. Giroletti \& Panessa 2009; Bontempi et al. 2012; Hada et al. 2013). What are the main elements that VLBI - and only VLBI - can bring in to the analysis of AGNs? Certainly, the possibility to obtain images of structures as small as, or even smaller than, one parsec. Moreover, since the linear scales become so small, it is possible to actually reveal, monitor, and study the time evolution of the 
finest AGN structures, with measurements of jet knot speed, which often appear to be faster than the speed of light; in turn, this provides constraints on the velocity and orientation of the AGN jet. Finally, thanks to the possibility to perform time and/or space resolved studies of the brightness temperature, spectral index, and polarimetry, we can infer the corresponding physical state, such as particle density, magnetic field structure, energy density. This provides fundamental clues to the understanding of the physics of the regions in the vicinity of SMBH, in both RL and RQ AGNs.

In this work, we briefly present the current VLBI arrays (Sect.2), and then focus on two cases in which VLBI monitoring campaigns have provided interesting clues on the AGN and jet physics: Mrk421 (in Sect. 3) and M87 (in Sect.4).

\section{VLBI arrays}

In this section, we list the currently operating VLBI arrays in the world. While a detailed description is not possible for every instrument, we focus on the peculiarities of each of them and provide references for a more detailed description in the web. Some more details are also given in Reid \& Honma (2013).

Located in the United States, the Very Long Baseline Array (VLBA) $\dagger$ is the first and largest largest full time, dedicated VLBI array. It consists of 10 identical $25 \mathrm{~m}$ stations, forming baselines up to $9000 \mathrm{~km}$, and equipped with large set of receivers $(0.3-86$ $\mathrm{GHz}$, some of which recently upgraded). It is a very flexible array, ideal for monitoring and multi-wavelength projects, such as the Monitoring Of Jets in AGN with VLBA Experiments (MOJAVE, Lister et al. 2009)

Starting from a core of European stations, the European VLBI Network (EVN) $\ddagger$ is a part-time network of otherwise independently operated telescopes now extending to Africa, Asia, and Puerto Rico. The network includes as many as 20 stations, each featuring different characteristics, so the array is somewhat complex to manage; on the other hand, it provides great sensitivity thanks to the presence of some very large aperture, soon to include the newly commissioned Sardinia Radio Telescope (64m diameter). Moreover, many stations are now connected with optical fibres and real time data transfer and correlation is routinely done.

Similar to the EVN, the Australian Long Baseline Array (LBA) 9 is a part-time network of stations in Oceania, ideal for observations in the southern hemisphere. Europeto-Australia real time observations have also been carried out with success (Giroletti et al. 2011). Other local arrays and networks exist, such as the Korean VLBI Network (KVN\|, observing up to $120 \mathrm{GHz}$ ), and the VLBI Exploration of Radio Astronomy (VERA ††, in Japan, with excellent astrometric capabilities).

Finally, the extreme angular resolution can be achieved through either short $\lambda$ or longer $B$. In the former scenario, we have the Global mm-VLBI Array (GMVA $\ddagger$ ) and the Event Horizon Telescope (EHT 9 ), while the latter extends to Space VLBI, such as that offered by the Russian-led Radioastron mission (Kovalev et al. 2014).

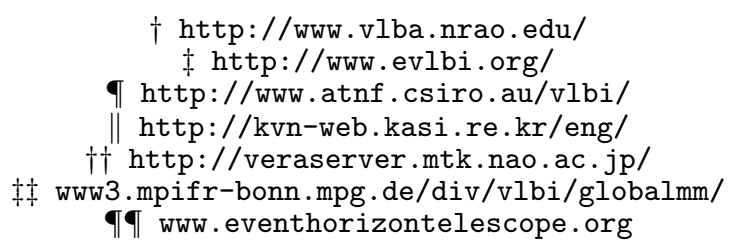




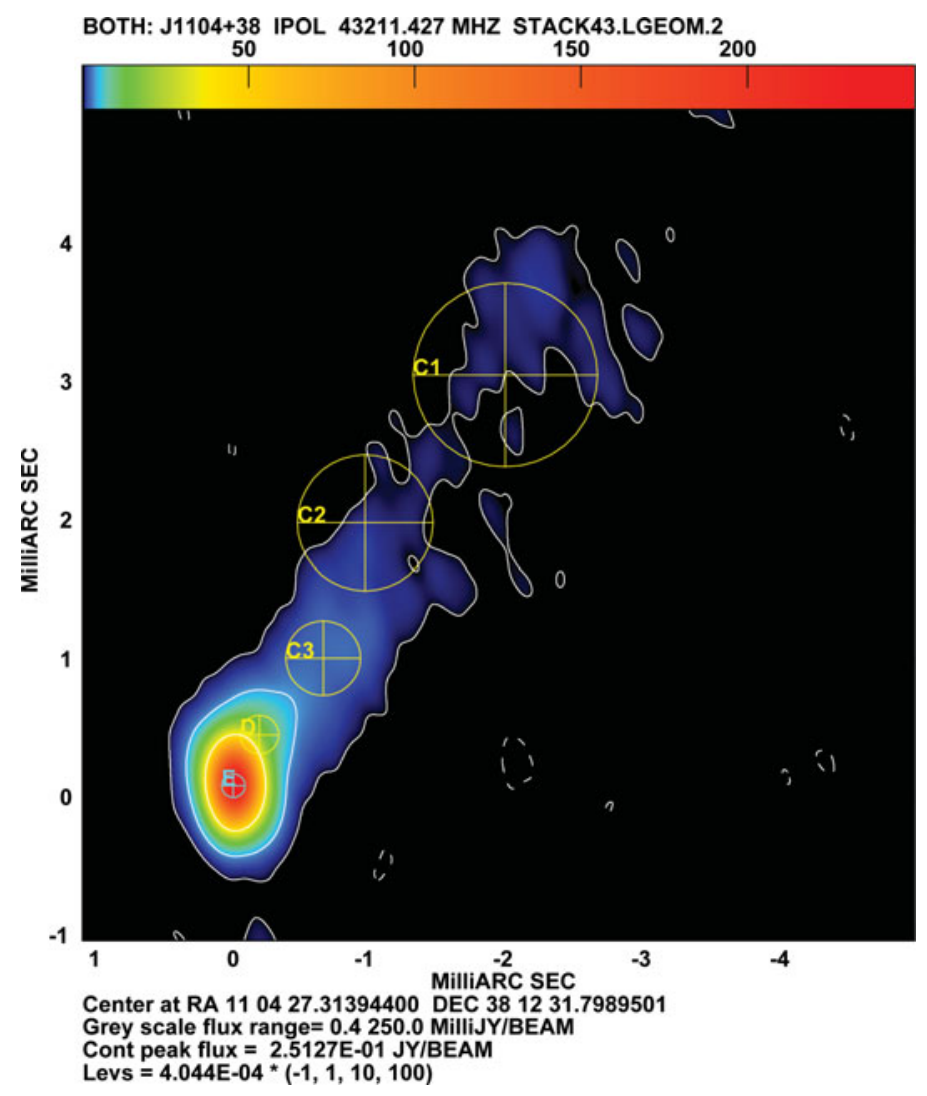

Figure 1. Average image of Mrk421 in 2011 based on the 12 observations at $43 \mathrm{GHz}$. Contours and color scale start at $0.4 \mathrm{mJy}_{\text {beam }}{ }^{-1}$. Overlaid circles show the Gaussian components fitting the visibility data.

\section{Mrk421 VLBA campaign}

The UV surveys conducted by Benjamin Markarian, of whom we celebrate the 100th anniversary at this conference, have revealed many different and remarkable types of galaxies. Among them, Markarian 421 (Mrk421, $z=0.031)$ is one of the most notable BL Lac objects, with radio power $P_{1.4}=10^{24.2} \mathrm{~W} \mathrm{~Hz}^{-1}$ and a spectral energy distribution typical of high synchrotron-peaked (HSP) blazars, with high energy emission extending up to the $\mathrm{TeV}$ regime. In this band, variability time scales as short as $\sim 1 \mathrm{hr}$ have been found (Gaidos et al. 1996), requiring the existence of a compact emission region, of a large Doppler factor $\delta$ and a small viewing angle $\theta$.

Mrk421 is also one of the brightest sources detected by the Fermi Large Area Telescope (LAT) in gamma rays (Abdo et al. 2009b). Actually, since the launch of Fermi, Mrk421 has almost continuously been subject to a world-wide MWL campaign (Abdo et al. 2011). VLBI is, however, the only technique capable of imaging the source on the linear scales implied by the MWL SED. For this reason, we organized a VLBA campaign throughout 2011, with one observation per month, at 15,24 , and $43 \mathrm{GHz}$, in total intensity and full polarization. In Lico et al. (2012) and Blasi et al. (2013), we reported the main results of the analysis of the total intensity images, which we summarize as follows:

- The source has a one-sided, core dominated structure at every frequency (see Fig. 1). The core peak brightness ranges between 150 and $400 \mathrm{mJy}^{\circ e a m^{-1}}$ and its size is as 


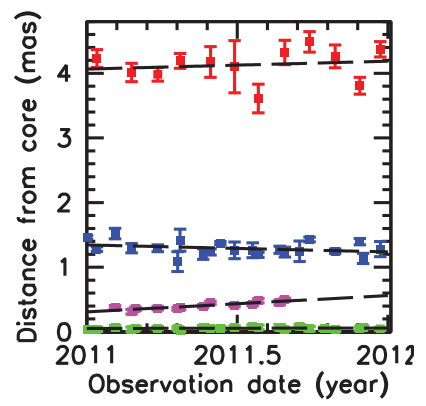

Figure 2. Distance from the core for the four components identified across epochs in the jet of Mrk421 (adapted from Blasi et al. 2013). The only one possibly displaying a significant proper

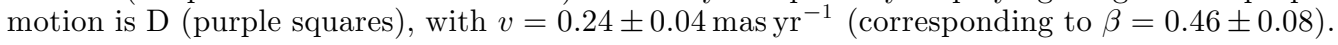

compact as $\sim 0.03 \mathrm{pc}($ at $43 \mathrm{GHz})$. The jet extends for a few parsecs in $\mathrm{PA}-34^{\circ}$, with a total flux of a few tens of milliJansky; no counterjet is detected down to $<0.1 \mathrm{mJy} \mathrm{beam}^{-1}$ level.

- A model fit to the visibilities provides a consistent description of the source structure in terms of a few Gaussian components, reasonably identified at the different frequencies. From a comparison of the images at the different epochs, we found no evidence of proper motion (see Fig. 2); in the few cases of a possible motion, the speed would be in any case not superluminal $(\beta \sim 0.5)$. This provides strong support for previous claims based on smaller datasets for BL Lacs (Piner \& Edwards 2005; Piner et al. 2010), in sharp contrast to the very large values of proper motion in quasars (up to $v \sim 30 c$, see e.g. Lister et al. 2009).

- Other observational values for the source are as follows: jet-counterjet ratio $R>$ 255 , image peak brightness temperature $T_{\mathrm{B}}>10^{11} \mathrm{~K}$, variability brightness temperature $T_{\mathrm{B} \text {,var }}>4 \times 10^{10} \mathrm{~K}$, observed core power $P_{c}=6.8 \times 10^{23.5} \mathrm{~W} \mathrm{~Hz}^{-1}$. These values constraint the Lorentz and Doppler factor obtained in the radio to much smaller values than those inferred in the high energy bands, suggesting the presence of a complex velocity structure in the jet of Mrk421. One possible scenario is that the jet is stratified, with a fast inner spine and a slow outer layer (Ghisellini et al. 2005), as also hinted at by the transversally resolved structure seen both in total intensity and in polarization; however, the latter feature could also be related to the presence of helical magnetic fields

- in polarization, the source shows two components: the core and the inner jet. The polarized intensity and the morphology are quite variable; in particular, the jet shows in some observations a transversally resolved structure with larger flux on the limbs. On the other hand, the electric vector position angle (EVPA) is remarkably stable in the inner jet, while it varies significantly in the core region. Also the optical EVPA is variable, with variations apparently connected to enhancements in the gamma-ray and radio core light curve (see Blasi et al. 2013, Lico et al. in prep. for further details)

\section{M87 EVN campaign}

M87 is one of the nearest radio galaxies $(d=16 \mathrm{Mpc})$, and probably our best laboratory for jet studies: its central black hole is as massive as $M_{\mathrm{BH}} \sim 6 \times 10^{9} M_{\odot}$, resulting in a superb linear resolution for VLBI studies $\left(1 \mathrm{mas}=0.081 \mathrm{pc}=140 R_{\mathrm{Schw}}\right)$. Moreover, it features prominent radio emission (mostly due to proximity, since the radio luminosity of 


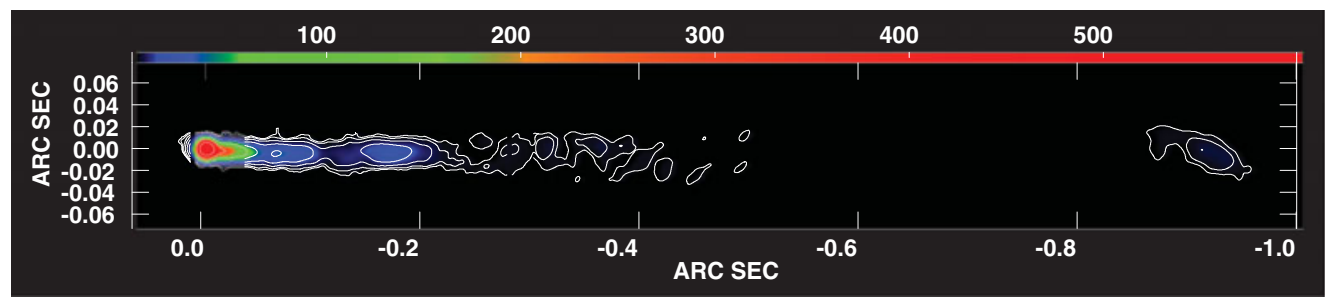

Figure 3. A stacked image of the M87 jet from our EVN observations at $5 \mathrm{GHz}$. The region at $\sim 900$ mas from the core is the extended HST-1 region, whose sub-structures are found to move with superluminal velocity during the time of our campaign. The lowest contours is traced at $0.3 \mathrm{mJy} \mathrm{beam}^{-1}$.

$P_{408 \mathrm{MHz}}=10^{25} \mathrm{~W} \mathrm{~Hz}^{-1}$ would make it a somewhat unremarkable low power FR1 radio galaxy), an extended optical and X-ray jet, and it has been detected in gamma rays. In the $\mathrm{MeV} / \mathrm{GeV}$ domain, Fermi has revealed it as a moderately bright, non variable source (Abdo et al. 2009a, 2010), while at Very High Energy (VHE, $E>100 \mathrm{GeV}$ ) it has shown several episodes of fast variability (Aharonian et al. 2006; Albert et al. 2008).

Two regions in the jet have been proposed as possible locations for the production of the highest energy emission: the radio compact core and the so called HST-1 jet knot some $70 \mathrm{pc}$ downstream the core. While the core is an obvious candidate because of its brightness and compactness, HST-1 was proposed on the basis of its large increase in radio, optical, and X-ray flux concurrent with the first detected VHE enhancement in 2005 (Harris et al. 2006); moreover, its morphology is characterized by the emergence of new sub-components that travel with superluminal velocity, a hallmark of Doppler beaming (Biretta et al. 1999; Cheung et al. 2007). However, HST-1 remained quite inactive during the 2008 VHE event, whereas the core showed a substantial $43 \mathrm{GHz}$ flux density increase at that time (Acciari et al. 2009).

Therefore, we started in late 2009 a monitoring project with the EVN in real time mode, providing ideal cadence, angular resolution, and sensitivity to monitor both the core and HST-1. At the most intense time of activity, we monitored the source every month (Giroletti et al. 2010), collecting a total of over 30 epochs between 2006 (including archival VLBA data) and the time of this conference. A sample image is shown in Fig. 3.

Enhanced VHE activity was reported in 2010 (Abramowski et al. 2012). Differently from the 2005 and 2008 events, no prominent increase in the radio flux density was revealed in either the core or HST-1 (but see Hada et al. 2012, for the discussion of a mild increase in the core light curve). However, we revealed a quite interesting morphological evolution within HST-1: while outer components continued their advance along the jet at a constant superluminal speed of $\sim 4 c$, a new feature appeared at the upstream edge soon after the VHE event. It then moved along the jet on an approximately radial path, with a velocity consistent with the other components (in the range $4 c-6 c$, with a relatively large uncertainty due to the lower number of epochs available to constrain the fit).

The tight constriaints on the superluminal speed observed in HST-1 posed by our observations suggest a Lorentz factor in the range $4 \leqslant \Gamma \leqslant 6$ and a Doppler factor $1.5 \leqslant \delta \leqslant 4$. A continuation of the coordinated VLBI and VHE monitoring is crucial to increase the number of events and find a coherent picture to understand these events. 


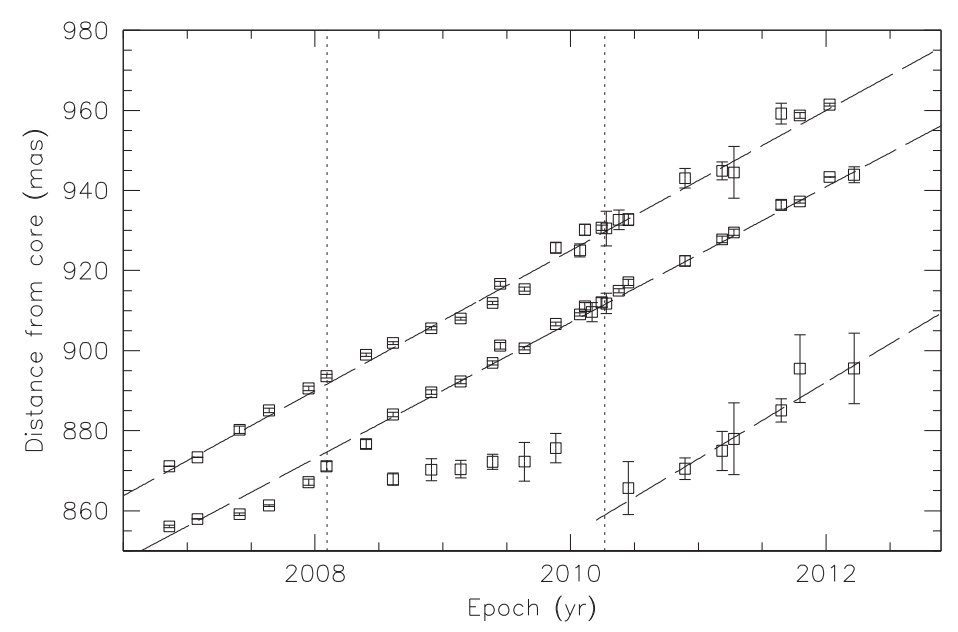

Figure 4. Motion of HST-1 subcomponents between 2006 and 2013. Empty squares show visibility model fit positions, dashed lines the linear best fit, and the vertical dotted lines the epochs of enhanced VHE activity. Adapted from Giroletti et al. (2012).

\section{Acknowledgements}

The meeting was a great experience, for which we owe our gratitude to the Local Organizing Committee. MG acknowledges support for his participation in the symposium from the International Astronomical Union and from the European Commission Seventh Framework Programme (FP/2007-2013) under grant agreement No 283393 (RadioNet3). The research leading to these results has received funding from grant PRIN-INAF-2011. The European VLBI Network is a joint facility of European, Chinese, South African and other radio astronomy institutes funded by their national research councils. The National Radio Astronomy Observatory is a facility of the National Science Foundation operated under cooperative agreement by Associated Universities, Inc.

\section{References}

Abdo, A. A., Ackermann, M., Ajello, M., et al. 2009b, ApJ, 700, 597

Abdo, A. A., Ackermann, M., Ajello, M., et al. 2009a, ApJ, 707, 55

Abdo, A. A., Ackermann, M., Ajello, M., et al. 2010, ApJ, 720, 912

Abdo, A. A., Ackermann, M., Ajello, M., et al. 2011, ApJ, 736, 131

Abramowski, A., Acero, F., Aharonian, F., et al. 2012, ApJ, 746, 151

Acciari, V. A., Aliu, E., Arlen, T., et al. 2009, Science, 325, 444

Aharonian, F., Akhperjanian, A. G., Bazer-Bachi, A. R., et al. 2006, Science, 314, 1424

Albert, J., Aliu, E., Anderhub, H., et al. 2008, ApJ, 685, L23

Biretta, J. A., Sparks, W. B., \& Macchetto, F. 1999, ApJ, 520, 621

Blasi, M. G., Lico, R., Giroletti, M., et al. 2013, A\&AA, 559, A75

Bontempi, P., Giroletti, M., Panessa, F., Orienti, M., \& Doi, A. 2012, MNRAS, 426, 588

Cheung, C. C., Harris, D. E., \& Stawarz, Ł. 2007, ApJ, 663, L65

Gaidos, J. A., Akerlof, C. W., Biller, S., et al. 1996, Natur, 383, 319

Ghisellini, G., Tavecchio, F., \& Chiaberge, M. 2005, A\& $A, 432,401$

Giroletti, M., Giovannini, G., Beilicke, M., et al. 2010, ATel, 2437, 1

Giroletti, M., Hada, K., Giovannini, G., et al. 2012, A\&A, 538, L10

Giroletti, M., Paragi, Z., Bignall, H., et al. 2011, A\&A, 528, L11

Giroletti, M. \& Panessa, F. 2009, ApJ, 706, L260 
Hada, K., Kino, M., \& Nagai, H., et al. 2012, ApJ, 760, 52

Hada, K., Doi, A., Nagai, H., et al. 2013, ApJ, 779, 6

Harris, D. E., Cheung, C. C., Biretta, J. A., et al. 2006, ApJ, 640, 211

Kovalev, Y., et al. these proceedings

Lico, R., Giroletti, M., \& Orienti, M., et al. 2012, A\&A, 545, A117

Lister, M. L., Aller, H. D., Aller, M. F., et al. 2009, AJ, 137, 3718

Piner, B. G., Pant, N., \& Edwards, P. G. 2010, ApJ, 723, 1150

Piner, B. G. \& Edwards, P. G. 2005, ApJ, 622, 168

Reid, M. J. \& Honma, M. 2013, arXiv, arXiv:1312.2871 